\title{
nab-Paclitaxel Plus Gemcitabine Versus Gemcitabine in Patients with Metastatic Pancreatic Adenocarcinoma: Canadian Subgroup Analysis of the Phase 3 MPACT Trial
}

\author{
Mustapha Tehfe $\cdot$ Scot Dowden · Hagen Kennecke · Robert El-Maraghi • \\ Bernard Lesperance · Felix Couture · Richard Letourneau · Helen Liu • \\ Alfredo Romano
}

Received: January 27, 2016/ Published online: April 16, 2016

(C) The Author(s) 2016. This article is published with open access at Springerlink.com

\section{ABSTRACT}

Introduction: The phase III MPACT trial in patients with metastatic pancreatic cancer (MPC) demonstrated superior efficacy of $n a b$-paclitaxel (nab-P) plus gemcitabine (Gem) compared with Gem monotherapy, including the primary endpoint of overall survival (OS; median 8.7 vs. 6.6 months; hazard ratio [HR] $0.72 ; \quad P<0.001)$. A significant treatment difference favoring nab-P + Gem over Gem was

Enhanced content To view enhanced content for this article go to http://www.medengine.com/Redeem/3E84 F06021D67FC0.

Electronic supplementary material The online version of this article (doi:10.1007/s12325-016-0327-4) contains supplementary material, which is available to authorized users.

M. Tehfe $(\bowtie) \cdot$ R. Letourneau

Centre hospitalier de l'université de Montréal

(CHUM), Montreal, QC, Canada

e-mail: mustapha.tehfe.chum@ssss.gouv.qc.ca

S. Dowden

Tom Baker Cancer Centre, Calgary, AB, Canada

H. Kennecke

British Columbia Cancer Agency, Vancouver, BC,

Canada

R. El-Maraghi

Royal Victoria Regional Health Centre, Barrie, ON, Canada observed for OS in patients treated in North America. The majority of patients were from the US (88\%) with only $12 \%$ from Canada. Healthcare systems and treatment patterns are different between the 2 countries, and there is limited published information on outcomes of Canadian patients treated with first-line $n a b-\mathrm{P}+\mathrm{Gem}$. This analysis evaluated efficacy and safety outcomes in Canadian patients in the MPACT trial.

Methods: Treatment-naive patients with MPC $(N=861)$ received either nab-P $125 \mathrm{mg} /$ $\mathrm{m}^{2}+$ Gem $1000 \mathrm{mg} / \mathrm{m}^{2}$ on days 1,8 , and 15 every 4 weeks or Gem $1000 \mathrm{mg} / \mathrm{m}^{2}$ weekly for the first 7 of 8 weeks (cycle 1) and then on days 1,8 , and 15 every 4 weeks (cycle $\geq 2$ ).

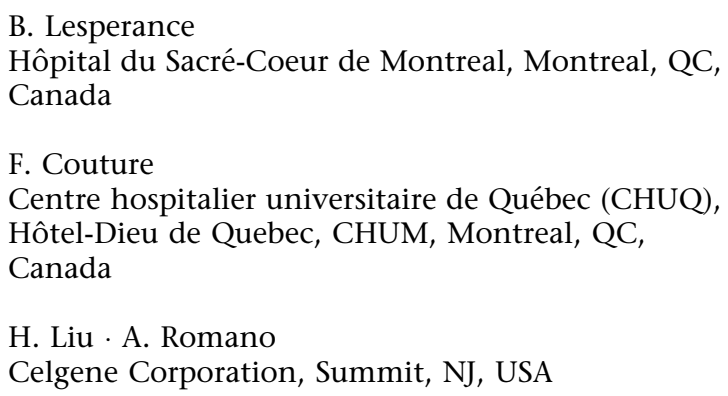


Results: The MPACT trial enrolled 63 patients in Canada. Baseline characteristics were well balanced and comparable with those of the intent-to-treat population. Both OS (median 11.9 vs. 7.1 months; HR $0.76 ; P=0.373)$ and progression-free survival (median 7.2 vs. 5.2 months; HR $0.65 ; \quad P=0.224$ ) were numerically longer and overall response rate $(27 \%$ vs. $17 \% ; \quad P=0.312)$ was numerically higher with nab-P + Gem vs. Gem. The most common grade $\geq 3$ adverse events with $n a b-\mathrm{P}+\mathrm{Gem}$ vs. Gem were neutropenia (22\% vs. $10 \%)$, fatigue $(34 \%$ vs. $33 \%)$, and neuropathy ( $25 \%$ vs. $0 \%$ ).

Conclusion: This subanalysis confirmed that $n a b-\mathrm{P}+\mathrm{Gem}$ is an efficacious treatment option and has a manageable safety profile in patients with MPC treated in Canada.

Trial registration: ClinicalTrials.gov identifier, NCT00844649.

Funding: Celgene Corporation, Summit, NJ, USA.

Keywords: Canada; Gemcitabine; Metastatic pancreatic cancer; MPACT; nab-Paclitaxel; Oncology; Subgroup analysis

\section{INTRODUCTION}

Pancreatic cancer (PC) is the fourth leading cause of cancer-related mortality in Canada, the US, and Europe [1-3]. Estimates suggested that in 2015, 4800 Canadians were diagnosed with PC and 4600 died from the disease [1]. The 5 -year relative survival ratio, which compares survival of patients with cancer to those without cancer, is $8 \%$ among Canadian patients with PC [1]. The observed 5-year survival rate for patients with PC is also $8 \%$, a statistic that has not improved in the last 40 years [4]. Risk factors for the development of PC include smoking and obesity in addition to several well-established genetic mutations [5].

Surgery is the only potentially curative treatment for PC; however, surgical resection is an option in only $17 \%$ vs. $19 \%$ of Canadian PC cases [6]. Patients who are not eligible for surgery may be treated with systemic cancer therapy. For many years, gemcitabine (Gem) has been a standard treatment for metastatic PC (MPC) in Canada [4]. In addition, erlotinib is approved in Canada for the treatment of MPC, and the combination regimen of leucovorin, 5-fluorouracil (5-FU), irinotecan, and oxaliplatin (FOLFIRINOX) has been approved in some provinces [4]. The most recent regimen to gain approval for the treatment of MPC is $n a b$-paclitaxel (nab-P) plus Gem [4, 7]. Since receiving this approval, $n a b-\mathrm{P}+\mathrm{Gem}$ has been evaluated by the pan-Canadian Oncology Drug Review [8] and has been deemed reimbursable by most but not all Canadian provinces [4].

Health Canada approved nab-P + Gem for the treatment of MPC based on the results of the phase III MPACT trial $(N=861$; ClinicalTrials.gov identifier, NCT00844649), which compared nab-P + Gem vs. Gem alone [7]. In the MPACT trial, nab-P + Gem demonstrated superior efficacy in all trial endpoints, including the primary endpoint of overall survival (OS; median 8.5 vs. 6.7 months; hazard ratio [HR] $0.72 ; P<0.001)$ [9]. An updated analysis revealed an even greater difference in median OS: 8.7 vs. 6.6 months (HR $0.72 ; P<0.001$ ) [10]. The combination of $n a b-\mathrm{P}+\mathrm{Gem}$ vs. Gem alone also demonstrated a longer progression-free survival (PFS; median 5.5 vs. 3.7 months; HR $0.69 ; P<0.001)$ and higher overall response rate (ORR) by both independent $(23 \%$ vs. $7 \% ; P<0.001)$ and investigator $(29 \%$ vs. $8 \% ; P<0.001)$ review [9]. Grade $\geq 3$ adverse events in the MPACT trial 
were effectively managed by dose reductions and treatment delays.

MPACT was a global trial that enrolled patients at 151 community and academic sites in North America (63\%), Eastern Europe (15\%), Australia (14\%), and Western Europe (9\%) [9]. Because cancer mortality rates vary among countries worldwide [11] (likely a reflection of differences in healthcare systems), clinical trials in oncology routinely stratify patient randomization by region. Geographic region was a stratification factor in the MPACT trial, as were performance status and presence or absence of liver metastases [9]. A significant treatment difference was observed for OS within the North American patient population, which consisted of patients primarily from the US $(88 \%)$ and a small subset from Canada (12\%). Additionally, a stepwise multivariate analysis revealed a lower risk of death for patients in North America, regardless of treatment [12]. Healthcare systems and treatment patterns are different between the US and Canada, and limited published information is available on the outcomes of Canadian patients treated with first-line $n a b-\mathrm{P}+\mathrm{Gem}$. To understand how $n a b-\mathrm{P}+\mathrm{Gem}$ compared with Gem alone in Canadian patients, a subanalysis of the MPACT trial was undertaken to compare efficacy, safety, and treatment exposure in the subgroup of patients treated in Canada.

\section{METHODS}

The MPACT trial was approved by the independent ethics committee at each participating institution and was conducted in accordance with the International Conference on Harmonisation E6 requirements for Good Clinical Practice [9]. All procedures followed were in accordance with the ethical standards of the responsible committee on human experimentation (institutional and national) and with the Helsinki Declaration of 1964, as revised in 2013. Informed consent was obtained from all patients for being included in the study.

\section{Patients}

Study design and patient characteristics for the phase III MPACT trial have been described previously [9]. Briefly, adults ( $\geq 18$ years of age) with a Karnofsky performance status (KPS) $\geq 70$, no prior chemotherapy received for metastatic disease, and histologically or cytologically confirmed MPC as assessed by Response Evaluation Criteria In Solid Tumors (RECIST; version 1.0) [13] were included in this analysis. Patients were permitted to have received 5-FU or Gem as a radiation sensitizer in the adjuvant setting, provided that treatment was received $\geq 6$ months prior to randomization. Adequate hematologic, hepatic, and renal function was also required.

\section{Study Design}

Patients were randomized $1: 1$ to receive (1) intravenous nab-P $125 \mathrm{mg} / \mathrm{m}^{2}$ followed by intravenous Gem $1000 \mathrm{mg} / \mathrm{m}^{2}$ on days 1, 8, $15,29,36$, and 43 of an 8 -week cycle (cycle 1 ), then on days 1,8 , and 15 every 4 weeks for each subsequent cycle or (2) Gem $1000 \mathrm{mg} / \mathrm{m}^{2}$ weekly for the first 7 weeks of an 8-week cycle (cycle 1), then for the first 3 weeks of a 4 -week cycle (cycle $\geq 2$ ). Patients were stratified according to KPS, presence of liver metastases, and geographic region. Patients were treated until either disease progression or unacceptable toxicity. Tumor response was evaluated every 8 weeks using spiral computed tomography or magnetic resonance imaging. At baseline and every 8 weeks thereafter, serial 
measurements of carbohydrate antigen 19-9 (CA 19-9) levels were obtained. The primary endpoint was OS, and secondary endpoints were PFS and ORR, as assessed by independent radiological review according to RECIST version 1.0. Also examined was $O S$ as a function of specific decreases in CA $19-9$ levels (20\% and $60 \%)$. Safety was graded by the National Cancer Institute Common Terminology Criteria for Adverse Events, version 3.0 [14], and summarized according to the Medical Dictionary for Regulatory Activities, version $15.0[15]$.

\section{Statistical Analyses}

Efficacy analyses were performed on all randomized patients in the Canadian cohort. The Kaplan-Meier method was used to determine OS, and statistical significance was assessed with a log-rank test. A stratified Cox proportional hazards model was used to determine the associated HR and two-sided 95\% confidence intervals (CIs). For patients who were lost to follow-up, survival data were censored at the last date that they were known to be alive. The original cutoff for OS analysis was September 17, 2012. A nonstratified log-rank test was PFS between the treatment arms, and the HR and two-sided 95\% CIs were estimated by a Cox proportional hazards model. Differences in ORR were assessed by $\chi^{2}$ test.

\section{RESULTS}

\section{Baseline Characteristics}

A total of 63 patients (33 receiving nab-P + Gem and 30 receiving Gem alone) were randomized for treatment at 7 Canadian centers (average number of patients per center was 4.7 for $n a b-\mathrm{P}+\mathrm{Gem}$ and 4.3 for Gem alone). In general, baseline characteristics were well balanced between the 2 treatment arms in the Canadian cohort and were similar to those of the intent-to-treat (ITT) population from the MPACT trial; however, some differences were noted (Table 1) [9]. The Canadian cohort included a greater percentage of male patients, patients with $\geq 3$ metastatic sites, and patients with a biliary stent compared with the ITT population. Fewer patients in the Canadian cohort had a KPS of 100 or previous Whipple procedure than in the ITT population. In the Canadian cohort, there was a greater percentage of patients whose primary tumor was located in the head of the pancreas in the nab-P + Gem arm compared with the Gem-alone arm.

\section{Efficacy}

\section{Overall Survival in the Canadian Population}

The OS data in the Canadian population were based on 47 deaths (75\% of the population), including 24 in the $n a b-\mathrm{P}+$ Gem arm (73\%) and 23 in the Gem-alone arm (77\%). In the Canadian cohort, OS was numerically longer with nab-P + Gem vs. Gem alone (median 11.9 vs. 7.1 months; HR 0.76; 95\% CI 0.41-1.40; $P=0.373$; Fig. 1). Kaplan-Meier estimates of OS at 24 months following randomization were $15 \%$ for $n a b-\mathrm{P}+\mathrm{Gem}$ and $9 \%$ for Gem alone.

\section{Progression-Free Survival}

For the PFS analysis by independent radiological review, 38 patients in the Canadian cohort (60\%) either had progressive disease or had died, including $23(70 \%)$ in the nab-P + Gem arm and 15 (50\%) in the Gem-alone arm. In the Canadian cohort, PFS was numerically longer in patients treated with $n a b-\mathrm{P}+\mathrm{Gem}$ vs. Gem alone (median 7.2 vs. 5.2 months; HR 0.65; 95\% CI 0.32-1.31; $P=0.224$; Table 2). 
Table 1 Baseline characteristics

\begin{tabular}{|c|c|c|c|}
\hline \multirow[t]{2}{*}{ Variable } & \multicolumn{2}{|l|}{ Patients in Canada } & \multirow{2}{*}{$\begin{array}{l}\text { ITT population [9] } \\
(N=861)\end{array}$} \\
\hline & $n a b-P+\operatorname{Gem}(n=33)$ & $\overline{\text { Gem }(n=30)}$ & \\
\hline Age, median (range), years & $61.0(34-77)$ & $61.5(49-72)$ & $63.0(27-88)$ \\
\hline \multicolumn{4}{|l|}{ Sex, $n(\%)$} \\
\hline Female & $12(36)$ & $9(30)$ & $359(42)$ \\
\hline Male & $21(64)$ & $21(70)$ & $502(58)$ \\
\hline \multicolumn{4}{|l|}{$\mathrm{KPS}, n(\%)$} \\
\hline 100 & $2(6)$ & $3(10)$ & $138(16)^{\mathrm{a}}$ \\
\hline 90 & $18(55)$ & $14(47)$ & $378(44)^{\mathrm{a}}$ \\
\hline 80 & $9(27)$ & $12(40)$ & $277(32)^{\mathrm{a}}$ \\
\hline 70 & $4(12)$ & $1(3)$ & $63(7)^{\mathrm{a}}$ \\
\hline 60 & 0 & 0 & $2(<1)^{\mathrm{a}}$ \\
\hline \multicolumn{4}{|c|}{ Number of metastatic sites, $n(\%)$} \\
\hline 1 & $4(12)$ & $1(3)$ & $54(6)$ \\
\hline 2 & $11(33)$ & $11(37)$ & $408(47)$ \\
\hline$\geq 3$ & $18(55)$ & $18(60)$ & $399(46)$ \\
\hline \multicolumn{4}{|l|}{ Sites of metastases, $n(\%)$} \\
\hline Liver & $27(82)$ & $25(83)$ & $725(84)$ \\
\hline Lung & $14(42)$ & $18(60)$ & $337(39)$ \\
\hline Peritoneum & $2(6)$ & $1(3)$ & $29(3)$ \\
\hline \multicolumn{4}{|c|}{ Pancreatic tumor location, $n(\%)$} \\
\hline Head & $18(55)$ & $9(30)$ & $371(43)$ \\
\hline Body & $11(33)$ & $11(37)$ & $268(31)$ \\
\hline Tail & $4(12)$ & $10(33)$ & $215(25)$ \\
\hline Unknown & 0 & 0 & $7(1)$ \\
\hline \multicolumn{4}{|l|}{ CA $19-9, n(\%)$} \\
\hline Normal & $3(9)$ & $3(10)$ & $116(15)^{b}$ \\
\hline$>\mathrm{ULN}$ and $<59 \times \mathrm{ULN}$ & $11(33)$ & $11(37)$ & $242(32)^{b}$ \\
\hline$\geq 59 \times \mathrm{ULN}$ & $16(48)$ & $11(37)$ & $392(52)^{b}$ \\
\hline Unknown & $3(9)$ & $5(17)$ & $111(13)^{c}$ \\
\hline Biliary stent, $n(\%)$ & $11(33)$ & $8(27)$ & $62(7)$ \\
\hline
\end{tabular}


Table 1 continued

\begin{tabular}{|c|c|c|c|}
\hline \multirow[t]{2}{*}{ Variable } & \multicolumn{2}{|l|}{ Patients in Canada } & \multirow{2}{*}{$\begin{array}{l}\text { ITT population [9] } \\
(N=861)\end{array}$} \\
\hline & $n a b-P+\operatorname{Gem}(n=33)$ & $\operatorname{Gem}(n=30)$ & \\
\hline Previous Whipple procedure, $n(\%)$ & $3(9)$ & $2(7)$ & $148(17)$ \\
\hline
\end{tabular}

CA 19-9 carbohydrate antigen 19-9, Gem gemcitabine, ITT intent-to-treat, KPS Karnofsky performance status, nab-P nab-paclitaxel, ULN upper limit of normal

a Total evaluable patients $=858$

b Total evaluable patients $=750$

c This value was not reported in Reference [9]

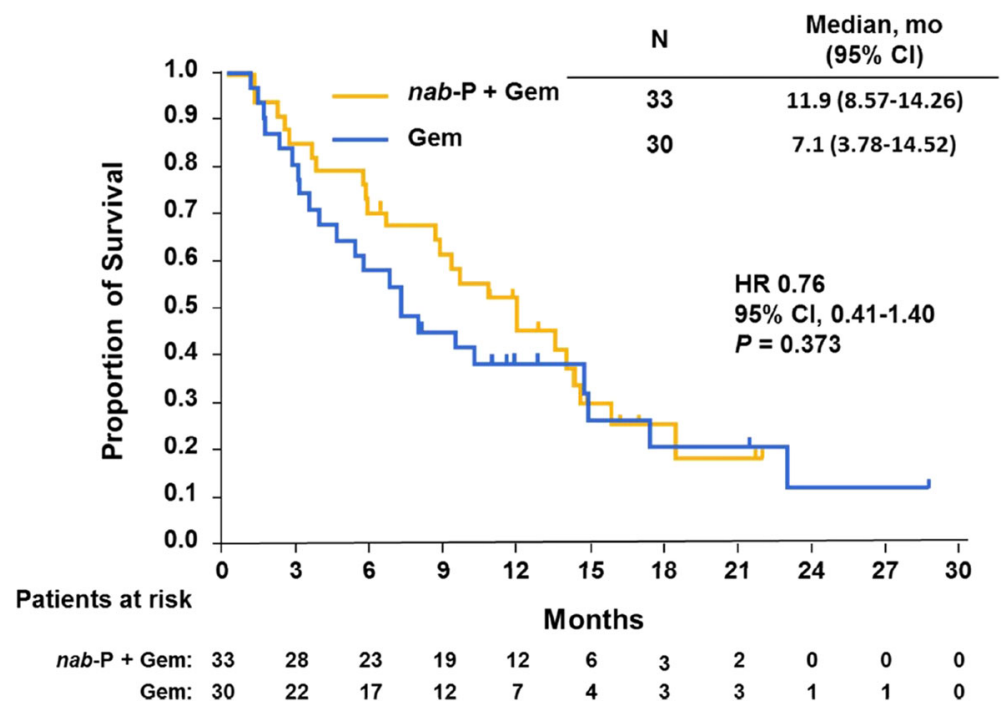

Fig. 1 Overall survival in Canadian patients. KaplanMeier curve of overall survival for patients in Canada treated with either nab-paclitaxel + gemcitabine or gemcitabine alone. $P$ value was generated from a log-rank test. $C I$ confidence interval, Gem gemcitabine, $H R$ hazard ratio, nab-P nab-paclitaxel

\section{Overall Response Rate}

The independently assessed ORR in the Canadian cohort was numerically higher in patients treated with nab-P + Gem vs. Gem alone ( $27 \%$ vs. $17 \%$; response rate ratio, 1.64 ; 95\% CI 0.62-4.34; $P=0.312$; Table 2). No Canadian patient in either treatment arm achieved a complete response. The disease control rate (partial response + stable disease [for $\geq 16$ weeks]) was also numerically higher in Canadian patients treated with $n a b-\mathrm{P}+\mathrm{Gem}$ than in patients treated with Gem alone $(58 \%$ vs. $37 \%$; response rate ratio, $1.57 ; P=0.097$ ).

\section{Evaluation of CA 19-9 Levels}

Patients in the Canadian cohort were evaluated for CA 19-9 levels at baseline and every 8 weeks. Assessment of OS in relation to CA 19-9 decrease (both 20\% and 60\%) was based on the 39 patients who had a measurement at baseline and $\geq 1$ time point after baseline. In a pooled analysis of these 39 patients (which included both treatment groups), OS was significantly longer in Canadian patients who had a $\geq 20 \%$ CA 19-9 decrease from baseline to nadir compared with those who had a $<20 \%$ decrease (median 14.5 vs. 7.8 months; HR 0.28; 
Table 2 PFS and ORR by independent radiological review in patients in Canada and the ITT population

\begin{tabular}{|c|c|c|c|c|}
\hline \multirow[t]{2}{*}{ Variable } & \multicolumn{2}{|l|}{ Patients in Canada } & \multicolumn{2}{|l|}{ ITT population [9] } \\
\hline & $n a b-\mathrm{P}+\mathrm{Gem}(n=33)$ & Gem $(n=30)$ & $n a b-\mathrm{P}+\mathrm{Gem}(n=431)$ & $\operatorname{Gem}(n=430)$ \\
\hline \multicolumn{5}{|l|}{ PFS } \\
\hline Median PFS, months & 7.2 & 5.2 & 5.5 & 3.7 \\
\hline HR (95\% CI) & $0.65(0.32-1.31)$ & & $0.69(0.58-0.82)$ & \\
\hline$P$ value & 0.224 & & $<0.001$ & \\
\hline \multicolumn{5}{|l|}{ ORR } \\
\hline ORR, $n(\%)$ & $9(27)$ & $5(17)$ & $99(23)$ & $31(7)$ \\
\hline RRR (95\% CI) & $1.64(0.62-4.34)$ & & $3.19(2.18-4.66)$ & \\
\hline$P$ value & 0.312 & & $<0.001$ & \\
\hline Complete response, $n(\%)$ & 0 & 0 & $1(<1)$ & 0 \\
\hline Partial response, $n(\%)$ & $9(27)$ & $5(17)$ & $98(23)$ & $31(7)$ \\
\hline Stable disease, $n(\%)$ & $10(30)$ & $8(27)$ & $118(27)$ & $122(28)$ \\
\hline Progressive disease, $n(\%)$ & $4(12)$ & $6(20)$ & $86(20)$ & $110(26)$ \\
\hline Not evaluable, $n(\%)^{\mathrm{a}}$ & $10(30)$ & $11(37)$ & $128(30)$ & $167(39)$ \\
\hline
\end{tabular}

$C I$ confidence interval, Gem gemcitabine $H R$ hazard ratio, ITT intent-to-treat, nab-P nab-paclitaxel, ORR overall response rate, $P F S$ progression-free survival, $R R R$ response rate ratio

${ }^{a}$ Includes patients who did not have a postbaseline assessment

95\% CI $\quad 0.12-0.68 ; \quad P=0.003 ; \quad$ Table S1). Similarly, OS was significantly longer in patients with a $\geq 60 \%$ CA $19-9$ decrease from baseline to nadir than in those with a $<60 \%$ decrease (median 15.7 vs. 9.2 months; HR 0.40; 95\% CI $0.17-0.91 ; P=0.025)$. The maximum absolute percentage reductions in CA 19-9 from baseline (median) were $87.3 \%$ and $78.2 \%$ for patients in the nab-P + Gem and Gem-alone arms, respectively.

\section{Treatment Exposure and Secondary Therapy Use}

In general, treatment duration was slightly longer, but cumulative doses were similar in the Canadian subgroup compared with the ITT population. The median treatment duration was 4.1 months (range 0.3-20.7) in patients treated with nab-P + Gem and 3.1 months (range 0.6-16.6) in patients treated with Gem alone (Table 3). In the $n a b-\mathrm{P}+\mathrm{Gem}$ arm, $50 \%$ of patients had $\geq 1$ nab-P dose reduction and 38\% had $\geq 1$ Gem dose reduction. Forty percent of patients in the Gem-alone arm had $\geq 1$ dose reduction. In the nab-P + Gem arm, the median percentage of per-protocol doses given was $75.0 \%$ and $81.2 \%$ for $n a b-\mathrm{P}$ and Gem, respectively. The median percentage of per-protocol dose given in the Gem-alone arm was $85.0 \%$. For patients treated with $n a b-\mathrm{P}+\mathrm{Gem}$, the median nab-P dose intensity was $68.4 \mathrm{mg} / \mathrm{m}^{2} /$ week, and the median Gem dose intensity was $627.8 \mathrm{mg} / \mathrm{m}^{2} /$ week. The median dose intensity for Gem alone was $667.3 \mathrm{mg} / \mathrm{m}^{2} /$ week.

Fewer patients in the $n a b-\mathrm{P}+\mathrm{Gem}$ arm received a subsequent therapy than in the Gem-alone arm (30\% vs. 43\%; Table 4). 
Table 3 Treatment exposure in patients in Canada and the treated population

\begin{tabular}{|c|c|c|c|c|}
\hline \multirow{2}{*}{$\begin{array}{l}\text { Treatment exposure and dose } \\
\text { modification }\end{array}$} & \multicolumn{2}{|l|}{ Patients in Canada } & \multicolumn{2}{|c|}{ All treated patients [9] } \\
\hline & $\begin{array}{l}n a b-\mathrm{P}+\mathrm{Gem} \\
(n=32)\end{array}$ & Gem $(n=30)$ & $\begin{array}{l}n a b-\mathrm{P}+\mathrm{Gem} \\
(n=421)\end{array}$ & Gem $(n=402)$ \\
\hline $\begin{array}{l}\text { Treatment duration, median (range), } \\
\text { months }\end{array}$ & $4.1(0.3-20.7)$ & $3.1(0.6-16.6)$ & $3.9(0.1-21.9)$ & $2.8(0.1-21.5)$ \\
\hline $\begin{array}{l}\text { No. of treatment cycles administered, } \\
\text { median (range) }\end{array}$ & $4.0(1-20)$ & $2.5(1-17)$ & $3.0(1-23)$ & $2.0(1-23)$ \\
\hline Cumulative nab-P dose, median, $\mathrm{mg} / \mathrm{m}^{2}$ & 1412.5 & - & 1425.0 & - \\
\hline Cumulative Gem dose, median, $\mathrm{mg} / \mathrm{m}^{2}$ & $12,000.0$ & 9800.0 & $11,400.0^{\mathrm{a}}$ & 9000.0 \\
\hline $\begin{array}{l}\text { Average nab-P dose intensity, median } \\
\text { (range) }, \mathrm{mg} / \mathrm{m}^{2} / \text { week }\end{array}$ & $68.4(15.6-95.5)$ & - & $74.13(15.6-96.0)$ & - \\
\hline $\begin{array}{l}\text { Average Gem dose intensity, median } \\
\text { (range), } \mathrm{mg} / \mathrm{m}^{2} / \text { week }\end{array}$ & $\begin{array}{l}627.8 \\
\quad(125.0-763.6)\end{array}$ & $\begin{array}{l}667.3 \\
\quad(125.0-875.0)\end{array}$ & $\begin{array}{l}597.3 \\
\quad(125.0-768.3)\end{array}$ & $\begin{array}{l}674.9 \\
\quad(123.6-907.4)\end{array}$ \\
\hline $\begin{array}{l}\text { Percentage of protocol nab-P dose, median } \\
\text { (range) }\end{array}$ & $75.0(16.7-100.0)$ & - & $80.6(16.7-100.0)$ & - \\
\hline $\begin{array}{l}\text { Percentage of protocol Gem dose, median } \\
\text { (range) }\end{array}$ & $81.2(14.3-96.8)$ & $\begin{array}{l}85.0 \\
\quad(14.3-100.0)\end{array}$ & $75.3(14.3-97.7)$ & $\begin{array}{l}84.6 \\
\quad(14.1-100.0)\end{array}$ \\
\hline $\begin{array}{l}\text { Patients with } \geq 1 \text { nab-P dose reduction, } \\
n(\%)\end{array}$ & $16(50)$ & - & $172(41)$ & - \\
\hline $\begin{array}{l}\text { Patients with } \geq 1 \text { Gem dose reduction, } \\
\quad n(\%)\end{array}$ & $12(38)$ & $12(40)$ & $198(47)$ & $132(33)$ \\
\hline $\begin{array}{l}\text { Patients with } \geq 1 \text { nab-P dose delay/not } \\
\text { given, } n(\%)\end{array}$ & $25(78)$ & - & $300(71)$ & - \\
\hline $\begin{array}{l}\text { Patients with } \geq 1 \mathrm{Gem} \text { dose delay/not given, } \\
n(\%)\end{array}$ & $23(72)$ & $20(67)$ & $295(70)$ & $230(57)$ \\
\hline
\end{tabular}

Gem Gemcitabine, nab-P nab-paclitaxel

a Total evaluable patients in the population $=420$

In both treatment arms, 5-FU- or capecitabine-based regimens were the most commonly used secondary therapies.

\section{Safety}

The most common grade $\geq 3$ nonhematologic adverse events were fatigue and peripheral neuropathy, which occurred in 34\% and 25\% of patients treated with $n a b-\mathrm{P}+\mathrm{Gem}$ and $33 \%$ and $0 \%$ of patients treated with Gem alone, respectively (Table 5). Neutropenia was the most common grade $\geq 3$ hematologic adverse event in both treatment arms (22\% for $n a b-\mathrm{P}+\mathrm{Gem}$ and $10 \%$ for Gem alone). The rates of grade $\geq 3$ anemia, thrombocytopenia, and leukopenia were similar between the 2 treatment arms. Grade $\geq 3$ vomiting was slightly more common in the Gem-alone arm vs. nab-P + Gem arm (13\% vs. $6 \%)$. 
Table 4 Subsequent therapy in patients in Canada and the ITT population

\begin{tabular}{|c|c|c|c|c|}
\hline \multirow[t]{2}{*}{ Subsequent therapy } & \multicolumn{2}{|c|}{ Patients in Canada } & \multicolumn{2}{|l|}{ ITT population } \\
\hline & $\begin{array}{l}n a b-\mathrm{P}+\mathrm{Gem} \\
(n=33)\end{array}$ & $\begin{array}{l}\text { Gem } \\
(n=30)\end{array}$ & $\begin{array}{l}n a b-\mathrm{P}+\mathrm{Gem} \\
(n=431)\end{array}$ & $\begin{array}{l}\text { Gem } \\
(n=430)\end{array}$ \\
\hline Patients with any subsequent therapy, $n$ (\%) & $10(30)$ & $13(43)$ & $162(38)$ & $179(42)$ \\
\hline 5-FU/Cape-based & $9(27)$ & $11(37)$ & $131(30)$ & $155(36)$ \\
\hline FOLFIRINOX (modified/unmodified) & $2(6)$ & $3(10)$ & $19(4)$ & $25(6)$ \\
\hline Other & $2(6)$ & $3(10)$ & $43(10)$ & $50(12)$ \\
\hline Erlotinib-based & $1(3)$ & 0 & $13(3)$ & $11(3)$ \\
\hline
\end{tabular}

5-FU 5-fluorouracil; Cape capecitabine; FOLFIRINOX leucovorin, 5-FU, irinotecan, oxaliplatin; Gem gemcitabine; ITT intent-to-treat; nab-P nab-paclitaxel

Table 5 Adverse events in patients in Canada and the treated population

\begin{tabular}{|c|c|c|c|c|}
\hline \multirow[t]{2}{*}{ Grade $\geq 3$ AEs } & \multicolumn{2}{|c|}{ Patients in Canada } & \multicolumn{2}{|c|}{ All treated patients [9] } \\
\hline & $\begin{array}{l}n a b-\mathrm{P}+\mathrm{Gem} \\
(n=32)\end{array}$ & $\begin{array}{l}\text { Gem } \\
(n=30)\end{array}$ & $\begin{array}{l}n a b-\mathrm{P}+\mathrm{Gem} \\
(n=421)\end{array}$ & $\begin{array}{l}\text { Gem } \\
(n=402)\end{array}$ \\
\hline Patients with at least 1 grade $\geq 3 \mathrm{AE}, n(\%)$ & $30(94)$ & $22(73)$ & NR & NR \\
\hline \multicolumn{5}{|l|}{ Hematologic AEs, $n(\%)$} \\
\hline Neutropenia & $7(22)$ & $3(10)$ & $153(38)^{\mathrm{a}}$ & $103(27)^{b}$ \\
\hline Anemia & $3(9)$ & $2(7)$ & $53(13)^{\mathrm{a}}$ & $48(12)^{b}$ \\
\hline Thrombocytopenia & $2(6)$ & $1(3)$ & $52(13)^{\mathrm{a}}$ & $36(9)^{b}$ \\
\hline Leukopenia & $1(3)$ & 0 & $124(31)^{\mathrm{a}}$ & $63(16)^{b}$ \\
\hline \multicolumn{5}{|l|}{ Nonhematologic AEs, $n$ (\%) } \\
\hline Fatigue & $11(34)$ & $10(33)$ & $70(17)$ & $27(7)$ \\
\hline Peripheral neuropathy $^{\mathrm{c}}$ & $8(25)$ & 0 & $70(17)$ & $3(1)$ \\
\hline Vomiting & $2(6)$ & $4(13)$ & NR & NR \\
\hline
\end{tabular}

$A E$ adverse event, Gem gemcitabine, nab-Pnab-paclitaxel, $N R$ not reported

a Total evaluable patients in the population $=405$

b Total evaluable patients in the population $=388$

c Grouped AE term

\section{DISCUSSION}

This subanalysis of the MPACT trial evaluated the efficacy and safety outcomes with $n a b-\mathrm{P}+\mathrm{Gem}$ vs. Gem alone in patients treated in Canada. The results in the Canadian cohort were consistent with those in the ITT population, in which $n a b-\mathrm{P}+\mathrm{Gem}$ was associated with improved OS, ORR, and PFS compared with Gem alone [9]. A survival difference of nearly 5 months was observed between the nab-P + Gem and Gem-alone arms in the Canadian subpopulation (median 11.9 vs. 7.1 months); however, this difference 
did not reach statistical significance. In addition, the $7 \%$ of patients treated in Canada had numerically longer OS and PFS and a higher ORR than patients globally in the ITT population, especially those receiving nab-P + Gem, although the outcomes could not be compared statistically given that the Canadian patients were a small subset and such a comparison was not planned in the trial protocol.

In general, treatment exposure and secondary therapy use were similar in the Canadian and ITT populations [9]; however, fewer Canadian patients treated with $n a b-\mathrm{P}+\mathrm{Gem}$ received a subsequent therapy than ITT patients treated with nab-P + Gem. Patients in the Canadian cohort had a lower incidence of hematologic adverse events than those in the ITT population, but Canadian patients had higher incidences of fatigue (both treatment arms) and peripheral neuropathy (nab-P + Gem only) than ITT patients.

An examination of baseline characteristics and treatment exposure did not reveal the reasons for the efficacy observed in the Canadian population. In fact, the Canadian cohort had higher rates of certain baseline characteristics that might be expected to be associated with worse efficacy, including the percentages of patients who were male, had $\geq 3$ sites of metastasis, or had a KPS $<100$ [11, 16-19]. Furthermore, as described herein, the treatment exposure in the Canadian cohort was similar to that in the ITT population, and the rates of secondary therapy use were similar or slightly lower than in the ITT population. Conversely, there was a higher rate of patients per treatment center in the Canadian cohort (63 patients $/ 7$ centers $=9$ patients/center) vs. the ITT population (861 patients $/ 151$ centers $=5.7$ patients/center), suggesting the possibility that study physicians in the Canadian cohort could have become more familiar with the per-protocol treatments. The potential effect of this familiarity on efficacy and tolerability is difficult to quantify.

In the MPACT trial, there was a lower risk of death for patients in North America, regardless of treatment. Whether unique features of the Canadian healthcare system might have influenced efficacy outcomes among patients in the MPACT trial is an interesting question. Canadian citizens have access to universal healthcare through the single-payer system; thus, all patients receive the same level of care. Universal healthcare may have allowed Canadian patients as a whole to receive more uniform access to medical visits, supportive care medications, and second-line therapies. The universal healthcare system also provides educational awareness programs and special care for those in need, including the elderly. The impact of these differences on treatment effectiveness at this point is speculative.

This subanalysis was subject to a number of limitations. This was an unplanned, post hoc analysis; no consideration in the study design was given to allowing for statistical comparisons within the cohort. In addition, this analysis included only 63 patients; this small sample size was likely the factor that prevented numerous treatment arm comparisons from reaching statistical significance. Finally, some differences were apparent between the treatment arms in the Canadian cohort of patients, including the higher percentage with tumors in the head of the pancreas in the $n a b-\mathrm{P}+\mathrm{Gem}$ arm vs. the Gem-alone arm at baseline and the higher percentage in the Gem-alone arm who received secondary therapies. These limitations must be kept in mind when interpreting the results of the study.

Health Canada has approved nab-P + Gem and FOLFIRINOX for the treatment of patients 
with MPC based on the phase III MPACT and PRODIGE trials (ClinicalTrials.gov identifier, NCT00112658), respectively, in which $n a b-\mathrm{P}+\mathrm{Gem}$ and FOLFIRINOX demonstrated superior efficacy vs. Gem alone [4, 9, 20]. However, there is little clinical evidence to guide treatment selection between these regimens. Direct comparisons across the trials are not appropriate given differences in patient populations. A recent retrospective analysis investigated the likelihood of meeting eligibility criteria for the MPACT and PRODIGE trials and possible associations with efficacy among patients with MPC who were treated with Gem at British Columbia regional cancer centers between January 2000 and December 2011 [21]. The analysis found that $45 \%$ and $25 \%$ of the analyzed cohort would have been eligible to receive $n a b-\mathrm{P}+\mathrm{Gem}$ and FOLFIRINOX, respectively. Performance status and elevated bilirubin level were the most frequent reasons for ineligibility regardless of whether MPACT or PRODIGE criteria were applied. Furthermore, the study suggested that simply meeting eligibility criteria had a dramatic impact on efficacy outcomes: even though all patients were treated with Gem, those who met the more stringent criteria of the PRODIGE trial had a longer median OS than those who met the more inclusive criteria of the MPACT trial (8.6 vs. 6.7 months). These data suggest a substantial correlation between eligibility criteria and efficacy, illustrating the importance of randomized trials for comparing treatments and avoiding cross-trial comparisons.

\section{CONCLUSIONS}

This subanalysis confirmed that $n a b-\mathrm{P}+\mathrm{Gem}$ is an efficacious treatment option for Canadian patients with MPC and has a manageable safety profile. The consistency of the findings in the overall treatment population lends support to their overall implications, despite certain limitations, such as the small sample size. Additional data on the efficacy and safety of $n a b-\mathrm{P}+\mathrm{Gem}$ in Canadian patients are likely to become increasingly available as physicians continue to use the regimen to treat their patients with MPC.

\section{ACKNOWLEDGMENTS}

Sponsorship for this study and article processing charges was funded by Celgene Corporation, Summit, NJ, United States. All named authors meet the International Committee of Medical Journal Editors (ICMJE) criteria for authorship of this manuscript, take responsibility for the integrity of the work as a whole, and have given final approval to the version to be published. Editorial assistance in the preparation of this manuscript was provided by Aaron Runkle, PhD, of MediTech Media. Support for this assistance was funded by Celgene Corporation. The authors were fully responsible for all content and editorial decisions of this manuscript.

Disclosures. Scot Dowden has participated in advisory boards as a speaker for Amgen, Bayer, Celgene, Lilly, and Roche. Hagen Kennecke has received honoraria and research support from Amgen and honoraria from Celgene and Novartis. Helen Liu is an employee and stockholder of Celgene. Robert El-Maraghi has participated in advisory boards as a speaker and has received honoraria from Celgene and Lilly, and has participated in advisory boards and received travel support and honoraria from Roche. Alfredo Romano is an employee and stockholder of Celgene. 
Mustapha Tehfe has participated in advisory boards for Celgene, Lilly, Ipsen, Merck, and Novartis and has been a speaker for Celgene, Lilly, Amgen, and Novartis. Bernard Lesperance, Felix Couture, and Richard Letourneau declare that they have no conflicts of interest.

Compliance with Ethics Guidelines. All procedures followed were in accordance with the ethical standards of the responsible committee on human experimentation (institutional and national) and with the Helsinki Declaration of 1964, as revised in 2013. Informed consent was obtained from all patients for being included in the study.

Open Access. This article is distributed under the terms of the Creative Commons Attribution-NonCommercial 4.0 International License (http://creativecommons.org/licenses/ by-nc/4.0/), which permits any noncommercial use, distribution, and reproduction in any medium, provided you give appropriate credit to the original author(s) and the source, provide a link to the Creative Commons license, and indicate if changes were made.

\section{REFERENCES}

1. Canadian Cancer Society. Canadian cancer statistics. 2015. Available at: http://www.cancer.ca/ /media/ cancer.ca/CW/cancer\%20information/cancer\%20101 /Canadian\%20cancer\%20statistics/Canadian-CancerStatistics-2015-EN.pdf. Accessed Nov 9, 2015.

2. American Cancer Society. Cancer facts and figures. 2015. Available at: http://www.cancer.org/ acs/groups/content/@editorial/documents/document/ acspc-044552.pdf. Accessed Nov 9, 2015.

3. Ferlay J, Steliarova-Foucher E, Lortet-Tieulent J, et al. Cancer incidence and mortality patterns in Europe: estimates for 40 countries in 2012. Eur J Cancer. 2013;49:1374-403.

4. Pancreatic Cancer Canada. Know the facts and statistics. Available at: http://www.pancreaticcancer
canada.ca/site/PageServer?pagename=facingpan creaticcancer_facts. Accessed Nov 9, 2015.

5. Chiorean EG, Coveler AL. Pancreatic cancer: optimizing treatment options, new, and emerging targeted therapies. Drug Des Devel Ther. 2015;9:3529-45.

6. Hurton S, MacDonald F, Porter G, Walsh M, Molinari M. The current state of pancreatic cancer in Canada: incidence, mortality, and surgical therapy. Pancreas. 2014;43:879-85.

7. Cancer Care Ontario. nab-Paclitaxel drug monograph. 2015.

8. Pan-Canadian Oncology Drug Review. Pan-Canadian oncology drug review final economic guidance report: nab-paclitaxel (Abraxane) for pancreatic cancer. Available at: https://www.cadth.ca/sites/default/files/pcodr/pcodrabraxane-mpc-fn-egr.pdf. Accessed Nov 9, 2015.

9. Von Hoff DD, Ervin T, Arena FP, et al. Increased survival in pancreatic cancer with nab-paclitaxel plus gemcitabine. N Engl J Med. 2013;369: 1691-703.

10. Goldstein D, El-Maraghi RH, Hammel $\mathrm{P}$, et al. Nab-paclitaxel plus gemcitabine for metastatic pancreatic cancer: long-term survival from a phase III trial. J Natl Cancer Inst. 2015;107:dju413.

11. World Health Organization. GLOBOCAN 2012: estimated cancer incidence, mortality and prevalence worldwide in 2012. Available at: http:// globocan.iarc.fr/Pages/fact_sheets_cancer.aspx. Accessed Sept 17, 2015.

12. Tabernero J, Chiorean EG, Infante JR, et al. Prognostic factors of survival in a randomized phase III trial (MPACT) of weekly nab-paclitaxel plus gemcitabine versus gemcitabine alone in patients with metastatic pancreatic cancer. Oncologist. 2015;20:143-50.

13. Therasse P, Arbuck SG, Eisenhauer EA, et al. New guidelines to evaluate the response to treatment in solid tumors. European Organization for Research and Treatment of Cancer, National Cancer Institute of the United States, National Cancer Institute of Canada. J Natl Cancer Inst. 2000;92:205-16.

14. Trotti A, Colevas AD, Setser A, et al. CTCAE v3.0: development of a comprehensive grading system for the adverse effects of cancer treatment. Semin Radiat Oncol. 2003;13:176-81.

15. MedDRA Maintenance and Support Services Organization. Introductory Guide to MedDRA Version 15.0. Chantily: MedDRA Maintenance and Support Services Organization; 2012. http:// 
www.meddra.org/sites/default/files/guidance/file/ intguide_15_0_english.pdf. Accessed Mar 24, 2016.

16. Kou T, Kanai M, Yamamoto M, et al. Prognostic model for survival based on readily available pretreatment factors in patients with advanced pancreatic cancer receiving palliative chemotherapy. Int J Clin Oncol. 2016;21:118-25.

17. Blank PR, Szucs TD. Pancreas cancer: influence of gender and other demographic and clinical parameters on survival [abstract 200]. J Clin Oncol. 2014;32(suppl 3).

18. Xue $\mathrm{P}, \mathrm{Zhu} \mathrm{L}$, Wan $\mathrm{Z}$, et al. A prognostic index model to predict the clinical outcomes for advanced pancreatic cancer patients following palliative chemotherapy. J Cancer Res Clin Oncol. 2015;141:1653-60.

19. Bolm L, Janssen S, Kasmann L, et al. Predicting survival after irradiation of metastases from pancreatic cancer. Anticancer Res. 2015;35:4105-8.

20. Conroy T, Desseigne $\mathrm{F}$, Ychou $\mathrm{M}$, et al. FOLFIRINOX versus gemcitabine for metastatic pancreatic cancer. N Engl J Med. 2011;364:1817-25.

21. Peixoto RD, Ho M, Renouf DJ, et al. Eligibility of metastatic pancreatic cancer patients for first-line palliative intent nab-paclitaxel plus gemcitabine versus FOLFIRINOX. Am J Clin Oncol. 2015. [Epub ahead of print]. 